\title{
Aarno Anaconat Avaliação do desempenho da Atenção Primária à Saúde em Florianópolis, Santa Catarina, 2012: estudo transversal de base populacional*
}

doi: 10.5123/S1679-49742018000400006

\section{Evaluation of Primary Health Care performance in Florianopolis, Santa Catarina, Brazil, 2012: a cross-sectional population-based study}

\author{
Evaluación del desempeño de la Atención Primaria a la Salud en el municipio de \\ Florianópolis, Santa Catarina, Brasil, 2012: estudio transversal de base poblacional
}

\author{
Tiago Barra Vidal' - (1) orcid.org/0000-0002-6103-9625 \\ Charles Dalcanale Tesser ${ }^{1}$ \\ Erno Harzheim² \\ Paulo Vinicius Nascimento Fontanive ${ }^{1}$ \\ 'Universidade Federal de Santa Catarina, Programa de Pós-Graduação em Saúde Coletiva, Florianópolis, SC, Brasil \\ ${ }^{2}$ Universidade Federal do Rio Grande do Sul, Programa de Pós-Graduação em Epidemiologia, Porto Alegre, RS, Brasil
}

\section{Resumo}

Objetivo: avaliar o desempenho da Atenção Primária à Saúde (APS) em Florianópolis, SC, Brasil. Métodos: estudo transversal de base populacional realizado com adultos residentes no território dos Centros de Saúde (CS) do Distrito Sanitário Norte do município em 2012; o instrumento Primary Care Assessment Tool (PCATool-Brasil) foi aplicado para avaliar a presença e a extensão dos atributos da APS. Resultados: dos 598 entrevistados, 68,4\% referiram utilizar os CS como serviço de referência; enquanto o Acesso de Primeiro Contato/subdimensão utilização foi 0 atributo melhor avaliado (8,4; $\left.\mathrm{IC}_{95 \%} 8,2 ; 8,6\right)$, o Acesso de Primeiro Contato/subdimensão acessibilidade foi o pior $\left(3,5 ; \mathrm{IC}_{95 \%} 3,3 ; 3,6\right)$; quatro $(36,6 \%)$ dos 11 CS avaliados apresentaram alto escore médio essencial e geral de APS, embora seu escore médio geral fosse baixo (6,4; $\mathrm{IC}_{95 \%}$ 6,2;6,5). Conclusão: considerando-se o modelo de APS avaliado pelo PCATool-Brasil, os serviços de APS necessitam ser aprimorados, especialmente nos componentes processo e estrutura.

Palavras-chave: Atenção Primária à Saúde; Estratégia Saúde da Família; Avaliação em Saúde; Estudos Transversais.

\footnotetext{
* Artigo derivado de tese de doutorado intitulada 'Modelos de agendamento e a relação com o desempenho da Atenção Primária à Saúde na percepção dos usuários no município de Florianópolis, SC', defendida por Tiago Barra Vidal junto ąo Programa de Pós-Graduação em Saúde Coletiva da Universidade Federal de Santa Catarina em 2018.
} 


\section{Introdução}

Avaliar a magnitude da Estratégia Saúde da Família (ESF) é uma tarefa para a qual o esforço coletivo é essencial, dada a heterogeneidade de suas ações no contexto nacional. Faz-se necessário um rigor metodológico na aplicação de instrumentos de avaliação da Atenção Primária à Saúde (APS), produção de conhecimento científico e busca de evidências da efetividade do modelo dessa atenção, visando à sua possível reorganização no país. ${ }^{1}$

Uma das formas de se avaliar a APS se encontra no instrumento Primary Care Assessment Tool (PCATool), criado para analisar o desempenho da APS pela presença e extensão de seus atributos essenciais (Acesso de Primeiro Contato, Integralidade, Longitudinalidade e Coordenação do Cuidado) e derivados (Orientação Familiar e Orientação Comunitária). Essa ferramenta de análise propõe questões sobre os serviços de saúde a serem respondidas por usuários adultos e crianças, gestores ou profissionais da área, conforme a versão adotada: para usuários adultos ou para crianças. Com base no modelo de avaliação da qualidade proposto por Donabedian, aspectos de estrutura, processo e resultados dos serviços de saúde são mensurados. ${ }^{1}$ Validado e utilizado em diferentes países, como Canadá, ${ }^{2}$ Estados Unidos, ${ }^{3,4}$ Espanha, ${ }^{5}$ China, ${ }^{6}$ Argentina ${ }^{7} \mathrm{e}$ Brasil,${ }^{8-10}$ o PCATool mostrou-se adequado em diferentes contextos sanitários e culturais.

\section{Uma das formas de se avaliar a APS se encontra no instrumento Primary Care Assessment Tool (PCATool), criado para analisar o desempenho da APS pela presença e extensão de seus atributos essenciais (Acesso de Primeiro Contato, Integralidade, Longitudinalidade e Coordenação do Cuidado) e derivados (Orientação Familiar e Orientação Comunitária).}

Mesmo nas capitais com experiências exitosas de APS, como Belo Horizonte, MG, Curitiba, PR, Rio de Janeiro, RJ, e Florianópolis, SC, a ESF apresenta componentes de estrutura e processo a serem aprimorados. Em Belo Horizonte, foram entrevistados os 147 gestores das unidades básicas de saúde (UBS) e o enfermeiro de cada uma das 538 equipes da ESF local. Os atributos mais bem avaliados por eles foram Acesso de Primeiro Contato/subdimensão utilização, Longitudinalidade e Integralidade; e os piores, Acesso de Primeiro Contato/ subdimensão acessibilidade e Orientação Comunitária, mostrando que as características de estrutura das UBS e de organização do processo de trabalho das equipes da ESF influenciam nesse desempenho. ${ }^{11}$

Em Curitiba, o instrumento PCATool-Brasil foi aplicado a 190 profissionais da ESF (91 médicos e 99 enfermeiros), nas UBS no município. 0 Acesso de Primeiro Contato/subdimensão acessibilidade foi o único atributo avaliado a obter baixo escore para APS, enquanto os demais alcançaram alto escore. ${ }^{12}$ No Rio de Janeiro, foram entrevistadas 2.710 pessoas com 0 instrumento PCATool-Brasil em sua versão para crianças, e 2.430 pessoas com a versão para adultos. As equipes da ESF na capital fluminense tiveram, em média, baixo escore geral de APS, sendo os piores desempenhos os dos atributos Integralidade e Acesso e Primeiro Contato/subdimensão acessibilidade; e os melhores escores, Acesso de Primeiro Contato/subdimensão utilização e Coordenação do Cuidado/subdimensão sistema de informações. ${ }^{13}$

A aplicabilidade do PCATool é variada, ao se observar a literatura internacional. No Canadá, Rowan et al. (2002) aplicaram o instrumento PCATool a 134 preceptores de Medicina de Família e Comunidade (MFC) para avaliar se eles seguiam os princípios da APS. 0 maior resultado foi encontrado para 0 atributo Coordenação do Cuidado, e o mais baixo para Competência Cultural. ${ }^{2}$ Tsai et al. (2010) entrevistaram 879 pessoas, entre crianças e adultos, para examinar a relação entre contar com um médico de referência e a qualidade das experiências ambulatoriais de cuidados médicos em Taiwan, onde existe uma cobertura de seguro de saúde universal: no arquipélago, o fato de ter um médico de referência mostrou-se significativamente associado com Acesso de Primeiro Contato/subdimensão utilização, Longitudinalidade, Orientação Familiar e Competência Cultural. ${ }^{6}$

Nos Estados Unidos, Clancy et al. (2007) avaliaram a percepção da atenção à saúde de usuários com diabetes mellitus de tipo 2 (DM2), no que se refere a consultas em grupo (grupo terapêutico). Aleatoriamente, foram designados 186 pacientes com DM2 descontrolado, para receberem cuidados individuais ou consultas em grupo durante 12 meses. Comparados com os pacientes que receberam consultas individuais, os pacientes atendidos 
em grupo apontaram maiores escores para os atributos Longitudinalidade $(\mathrm{p}=0,001)$, Orientação Comunitária $(\mathrm{p}<0,0001)$ e Competência Cultural $(\mathrm{p}=0,022) .{ }^{4}$

Florianópolis, há tempos, é reconhecida pela qualidade e cobertura da APS. Reconhecida com o terceiro melhor índice de desempenho do SUS (IDSUS) em 2012, ${ }^{14}$ sua APS é centrada na Estratégia Saúde da Família; ${ }^{15}$ porém, até a publicação deste artigo, não havia nenhuma avaliação de seu desempenho pelo PCATool-Brasil.

0 presente estudo teve como objetivo avaliar 0 desempenho da APS no município de Florianópolis, a partir da experiência de seus usuários adultos.

\section{Métodos}

Estudo transversal, realizado no Distrito Sanitário Norte (DSN) do município de Florianópolis em 2012, utilizando-se do PCATool-Brasil como instrumento de medida de desempenho da APS.

Florianópolis é a capital do estado de Santa Catarina. Localizada na região Sul do Brasil, a cidade contava com 421.240 habitantes em $2010 .{ }^{16}$ Sua rede municipal de aten- ção à saúde se organiza em cinco distritos sanitários (Figura 1) para atender a toda a sua população, da qual 64.732 eram adultos cobertos pelo DSN no período avaliado. ${ }^{17}$

Inicialmente, foram incluídos no estudo os usuários dos serviços de APS com 18 anos ou mais de idade residentes nos domicílios localizados no território de abrangência dos 11 Centros de Saúde (CS) do DSN.

Para o cálculo do tamanho da amostra, foi utilizado o software OpenEpi®. Verificou-se a necessidade da aplicação de 459 questionários. 0 percentual de perda acrescido à amostra estimada foi de 30\%, pelo que 598 questionários foram aplicados. Os parâmetros utilizados para esse cálculo foram: (i) nível de confiança de $95 \%$, (ii) precisão absoluta de 5\% e (iii) efeito de desenho (design effect: Deff) de 1,2 para o ajuste do efeito de cluster, estimando-se uma proporção de $50 \%$ de usuários que atribuiriam alto escore $(\geq 6,6)$ de APS para os serviços avaliados. Foram excluídos do estudo os entrevistados que não tinham como serviço de referência um dos CS em análise $(n=189)$.

A amostra dos domicílios foi definida a partir de conglomerados (clusters), estratificados por Centro

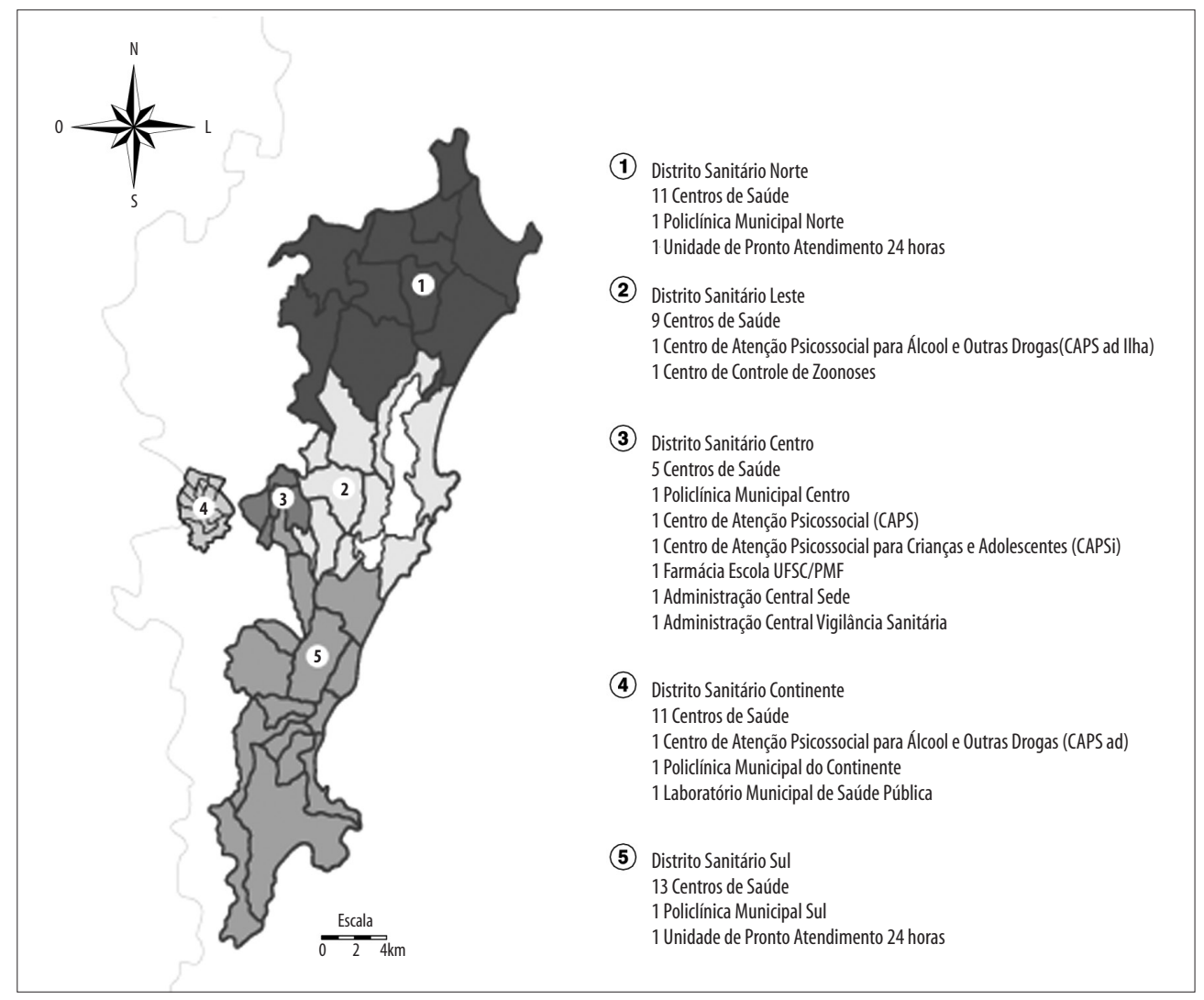

Figura 1 - Caracterização dos cinco Distritos Sanitários de Saúde de Florianópolis, Santa Catarina, 2012 
de Saúde e distribuídos proporcionalmente ao tamanho das respectivas populações adstritas. A seleção dos domicílios para aplicação do instrumento foi realizada mediante amostragem sistemática, por rua e casa. Primeiramente foram alocados os $11 \mathrm{CS}$, com os nomes das ruas de suas áreas de abrangência em uma planilha do aplicativo Microsoft Office Excel 2010®, em ordem alfabética. Logo, utilizou-se uma função para realocar na planilha, aleatoriamente, a ordem das ruas das áreas de abrangência de cada CS e determinar em que rua seriam iniciadas as entrevistas.

Para determinar de qual lado da rua teria início a seleção das casas onde as pessoas seriam entrevistadas, jogou-se uma moeda: segundo o lado sorteado, iniciava-se a busca pela primeira casa, passando-se à segunda casa do lado oposto, em seguida para a terceira casa do lado sorteado - ou seja, em zigue-zague -, até se alcançar o fim da rua. Somente após a conclusão das entrevistas de uma rua, partia-se para a próxima na ordem da lista, e assim consecutivamente, até tentar completar o número de entrevistas previsto para cada CS conforme descrito anteriormente, visando diminuir ao máximo o número de perdas na meta estimada.

Quando o entrevistador chegava ao domicílio sorteado, poderiam responder ao questionário, no máximo, dois adultos por moradia, limitando-se dessa forma 0 número de adultos por domicílio. Caso houvesse mais de dois adultos em um mesmo domicílio, respondiam ao questionário aqueles com maior idade.

Como entrevistadores, agentes comunitários de saúde (ACS) ( $\mathrm{n}=83$ ) foram encarregados e treinados para realizar esse trabalho sob a supervisão dos enfermeiros $(n=24)$ das equipes da ESF, norteadora da atenção à saúde em Florianópolis. ${ }^{15}$ A coleta de campo teve duração de cinco meses (maio-setembro/2012).

Com o objetivo de minimizar vieses de aferição, os ACSs não realizavam entrevistas em sua área de atuação, sendo alocados para outro CS do mesmo DSN. Realizou-se um estudo-piloto para o treinamento da equipe e esclarecimento de dúvidas quanto à aplicação do questionário.

A população estudada foi caracterizada por sexo, idade, cor da pele/raça e serviço de referência utilizado para cuidados em saúde - Centro de Saúde, unidade de pronto atendimento (UPA), policlínica municipal ou unidade da Saúde Suplementar (rede privada).

Conforme foi mencionado neste relato, o instrumento PCATool-Brasil mede a presença e extensão dos atributos da APS. Suas respostas são de tipo Likert, com escala de valores de 1 (Com certeza não) a 4 (Com certeza sim) e a opção adicional de numero 9: Não sei/não lembro. 0 PCATool-Brasil dispõe de 87 itens, divididos em dez componentes relacionados aos atributos essenciais e derivados da APS, elencados a seguir.

Primeiramente, os atributos essenciais:

(I) Grau de Afiliação com o Serviço de Saúde, identifica o serviço de referência dos cuidados em saúde do adulto entrevistado;

(II) Acesso de Primeiro Contato/subdimensão utilização, observa se o serviço de saúde é o primeiro a ser procurado quando ocorre um problema de saúde ou se o serviço avaliado é o único meio de encaminhamento para serviços especializados de saúde;

(III) Acesso de Primeiro Contato/subdimensão acessibilidade, procura avaliar a capacidade do serviço de saúde em atender seus usuários com agilidade quando apresentam um problema de saúde, além do tempo de espera até o paciente ser atendido pelo médico ou enfermeiro;

(IV) Longitudinalidade, verifica se o paciente se sente confortável para se expressar nas consultas, e se o médico ou enfermeiro conhece sua realidade biopsicossocial de forma ampla e integral;

(V) Coordenação do Cuidado/subdimensão integração de cuidados, remete à última consulta com especialista ou último serviço especializado acessado;

(VI) Coordenação do Cuidado/subdimensão sistema de informações, identifica se, quando vai ao serviço de saúde, o usuário é obrigado a apresentar algum registro de saúde ou boletim de atendimento prévio;

(VII) Integralidade/subdimensão serviços disponíveis, verifica se o entrevistado conhece os serviços disponíveis em seu estabelecimento de saúde, independentemente de ter utilizado esses serviços; $\mathrm{e}$

(VIII) Integralidade/subdimensão serviços prestados, identifica se alguns assuntos de saúde foram abordados com o entrevistado em sua última consulta (envelhecimento, por exemplo).

Atributos derivados:

(IX) Orientação Familiar, avalia se o usuário participa das decisões sobre seu tratamento ou de seus familiares, se em sua consulta são abordados problemas relacionados com sua dinâmica familiar; $\mathrm{e}$

(X) Orientação Comunitária, analisa de que forma o serviço de saúde reconhece os problemas de saúde da comunidade e estimula sua participação para resolvê-los. ${ }^{1}$ 
De acordo com o instrumento de validação do PCATool-Brasil em sua versão para adultos, os escores são padronizados por uma escala de valores de 0 a 10 , sendo os valores iguais ou superiores a 6,6 considerados como alto escore - 0 que corresponde a respostas para as opções 3 ou 4 na escala original do instrumento. A padronização para a escala 0-10 é feita da seguinte forma: ${ }^{8}$

$$
\text { Escore padronizado }=\frac{(\text { Escore }-1)}{(4-1)} \times 10
$$

Para a digitação e análise estatística dos dados, utilizou-se o software Microsoft Excel ${ }^{\circledR}$ 2010. A análise descritiva foi realizada apresentando-se a frequência absoluta, o percentual e a média.

0 cálculo do escore de desempenho da APS seguiu o manual do PCATool-Brasil do Ministério da Saúde. ${ }^{1}$ Foi calculado o escore da APS para cada um dos atributos, além do escore essencial (EE: resultado da média entre os escores dos atributos essenciais e o grau de afiliação), escore derivado (obtido pela média dos atributos derivados) e escore médio geral (EG: resultado da média entre todos os atributos [essenciais e derivados] e o grau de afiliação).

A participação dos entrevistados na pesquisa foi voluntária, mediante a assinatura de um Termo de Consentimento Livre e Esclarecido, conforme recomenda a Resolução do Conselho Nacional de Saúde (CNS) $\mathrm{n}^{0} 466$, de 12 de dezembro de 2012. A Comissão de Acompanhamento de Projetos de Pesquisa em Saúde de Florianópolis e o Comitê de Ética em Pesquisa com Seres Humanos da Universidade Federal de Santa Catarina (Parecer $\left.n^{\circ} 1.635 .663\right)$ aprovaram o projeto do estudo em 12 de junho de 2016.

\section{Resultados}

Foram entrevistados 598 usuários. Devido à metodologia utilizada, não houve perdas: 0 número de entrevistas realizadas previsto para cada Centro de Saúde foi atingido. Também não houve recusa por parte dos participantes. Os indivíduos impossibilitados de responder à entrevista no momento predeterminado foram agendados para outro dia de encontro com o entrevistador. A proporção de usuários que atribuíram alto escore de APS para os serviços avaliados foi de $46,4 \%(n=190)$. A média de idade das pessoas entrevistadas foi de 47 anos $(\mathrm{DP}=0,86)$, com maior proporção de mulheres $(72,6 \%) ; 92 \%$ das pessoas referiram ser de cor da pele/raça branca. A grande maioria dos entrevistados $(68,4 \% ; n=409)$ relataram utilizar os Centros de Saúde como serviço de referência, sendo esta $(n=409)$ a amostra das análises subsequentes. Outros dois serviços públicos foram mencionados como referência no DSN: a UPA $(9,7 \% ; n=57)$ e a policlínica municipal $(2,2 \% ; n=13)$; os demais entrevistados reportaram como referência uma unidade da Saúde Suplementar (19,9\%; n=119).

0 escore geral médio de APS foi, em geral, baixo $(\mathrm{EG}=6,4)$. A Tabela 1 apresenta os escores médios dos atributos e dos escores essencial, derivado e geral de APS, assim como a frequência de alto escore $(\geq 6,6)$ na avaliação dos usuários dos CS do DSN em 2012. 0 maior escore de APS avaliado correspondeu ao atributo Acesso de Primeiro Contato/subdimensão utilização $\left(8,4 ; \mathrm{IC}_{95 \%}\right.$ 8,2;8,6); o Acesso de Primeiro Contato/subdimensão acessibilidade foi o pior $\left(3,5 ; \mathrm{IC}_{95 \%} 3,3 ; 3,6\right)$.

As Figuras 2 e 3 apresentam, respectivamente, os escores médios essencial e geral do PCATool-Brasil entre todos os 11 Centros de Saúde do DSN. Observa-se que os mesmos quatro serviços avaliados (36,6\%) apresentaram alto escore de APS, tanto do escore médio essencial quanto do geral.

\section{Discussão}

Entre os centros de saúde do Distrito Sanitário Norte de Florianópolis, SC, observou-se heterogeneidade na avaliação do escore geral da Atenção Primária à Saúde e de cada um de seus atributos individualmente.

Os resultados encontrados são similares aos da revisão sistemática de Prates et al. sobre 22 artigos publicados na literatura nacional e internacional entre os anos de 2007 e 2015, que avaliaram o desempenho dos serviços de APS pelo instrumento PCATool na perspectiva do usuário. De acordo com a revisão citada, o atributo mais bem avaliado foi o Acesso de Primeiro Contato/subdimensão utilização (EG 3,5; $\left.\mathrm{IC}_{95 \%} 3,3 ; 3,6\right)$, e os piores, Acesso de Primeiro Contato/subdimensão acessibilidade, Integralidade, Orientação Familiar e Orientação Comunitária. ${ }^{18}$ No presente estudo, 0 atributo Acesso de Primeiro Contato apresentou uma variabilidade importante: enquanto a subdimensão acessibilidade obteve 0 pior escore, a subdimensão utilização apresentou o melhor (EG 8,41; $\left.\mathrm{IC}_{95 \%} 8,22 ; 8,61\right)$, isto entre todos os atributos avaliados. ${ }^{18}$ Essa variação também é encontrada em outras 
Tabela 1 - Escores médios dos atributos e dos escores essencial do PCATool-Brasil, a derivado e geral de Atenção Primária à Saúde, e frequência de alto escore na avaliação dos usuários $(n=409)$ dos Centros de Saúde do Distrito Sanitário Norte, Florianópolis, Santa Catarina, 2012

\begin{tabular}{lcr}
\hline Atributos da Atenção Primária à Saúde & $\begin{array}{c}\text { Escores médios } \\
\left(\text { IC }_{95 \%}{ }^{\mathbf{b}}\right.\end{array}$ & $\begin{array}{c}\text { Proporção de alto escore }(\geq 6,6) \\
\mathbf{n}(\%)\end{array}$ \\
\hline Acesso de Primeiro Contato - utilização & $8,4(8,2 ; 8,6)$ & $360(88,0)$ \\
Acesso de Primeiro Contato - acessibilidade & $3,5(3,3 ; 3,6)$ & $14(3,4)$ \\
Longitudinalidade & $6,4(6,2 ; 6,6)$ & $211(51,6)$ \\
Coordenação do Cuidado - integração de cuidados & $6,7(6,5 ; 7,0)$ & $151(36,9)$ \\
Coordenação do Cuidado - sistema de informações & $7,0(6,8 ; 7,2)$ & $287(70,2)$ \\
Integralidade - serviços disponíveis & $5,4(5,2 ; 5,7)$ & $142(34,7)$ \\
Integralidade - serviços prestados & $5,1(4,9 ; 5,3)$ & $125(30,6)$ \\
Escore essencial & $6,6(6,4 ; 6,7)$ & $208(50,9)$ \\
Orientação Familiar & $5,8(5,5 ; 6,1)$ & $205(50,1)$ \\
Orientação Comunitária & $5,2(4,9 ; 5,5)$ & $130(31,8)$ \\
Escore derivado & $5,5(5,2 ; 5,5)$ & $168(41,1)$ \\
Escore geral & $6,4(6,2 ; 6,5)$ & $190(46,4)$ \\
\hline
\end{tabular}

a) PCATool:Primary Care Assessment Tool.

b) IC ${ }_{95 \%}$ : intervalo de confiança de $95 \%$.

Nota:

Os escores assumem valores de 0 a 10

pesquisas que utilizaram o PCATool-Brasil. Araújo et al., em sua avaliação do desempenho da APS na perspectiva do idoso, entrevistaram 100 idosos atendidos por dez equipes de ESF das 20 UBS de Macaíba, RN, e obtiveram resultados semelhantes (utilização: $\mathrm{EG} 8,5$; $\left.\mathrm{IC}_{95 \%} 8,1 ; 9,0\right)$ (acessibilidade: $\left.\mathrm{EG} 3,8 ; \mathrm{IC}_{95 \%} 3,6 ; 4,1\right) .{ }^{19}$ Silva e Fracolli encontraram a mesma variabilidade ao entrevistarem 527 adultos acima de 18 anos, cadastrados em 33 unidades da ESF em Alfenas, MG (utilização: EG 8,6; DP 2,0) (acessibilidade: EG 3,2; DP 1,5). ${ }^{20}$

Esses resultados sugerem que, apesar de as pessoas utilizarem a ESF, há dificuldades relacionadas à acessibilidade desses serviços. Acesso não significa apenas a entrada do usuário no sistema de saúde ou a disponibilidade de serviços e recursos em determinado tempo e espaço, e sim o ajuste entre as necessidades da população e a presente oferta desses serviços. ${ }^{21}$

Entre os achados deste trabalho, a Longitudinalidade obteve baixo escore médio para APS, embora próximo do ponto de corte de 6,6 (EG 6,4; $\mathrm{IC}_{95 \%}$ $6,2 ; 6,6)$, resultado semelhante ao encontrado por Chomatas et al. em Curitiba (EG 6,6; $\left.\mathrm{IC}_{95 \%} 6,4 ; 6,7\right) .{ }^{12}$ Esse atributo é característica central e exclusiva da APS. Uma maior extensão da Longitudinalidade tende a produzir maior precisão diagnóstica e terapêutica, diminuindo o número de encaminhamentos desne- cessários, a realização de procedimentos de maior complexidade e, por conseguinte, menores custos para a Saúde Pública. ${ }^{22}$

0 atributo Coordenação do Cuidado obteve alto escore médio para APS, tanto na integração de cuidados (EG 6,75; IC ${ }_{95 \%} 6,5 ; 7,0$ ) como no sistema de informações ( $\left.\mathrm{EG} 7,0 ; \mathrm{IC}_{95 \%} 6,8 ; 7,2\right)$. À mesma conclusão chegaram Furtado et al. em estudo no qual aplicaram o PCATool-Brasil para 44 mães de crianças menores de 1 ano usuárias da ESF de Ribeirão Preto, SP: utilizando-se da escala original do instrumento, que varia de $1 \mathrm{a}$ 4, os valores acima de 3,0 por eles encontrados representam forte presença e extensão do atributo Coordenação do Cuidado, em suas subdimensões integração de cuidados (EG 3,7; DP 0,4) e sistema de informações (EG 3,6; DP 0,3). ${ }^{23}$ Isto mostra a capacidade das equipes da ESF em garantir a continuidade da atenção, no interior da rede de atenção à saúde. A disponibilidade da informação sobre o usuário na rede de serviços, ou seja, a existência de um prontuário com suas informações em todos os pontos da rede, é essencial para a coordenação da atenção à saúde. 0 envelhecimento populacional e o consequente aumento da prevalência das doenças crônicas tornam a integração do sistema de informações cada vez mais necessária. ${ }^{24}$ 


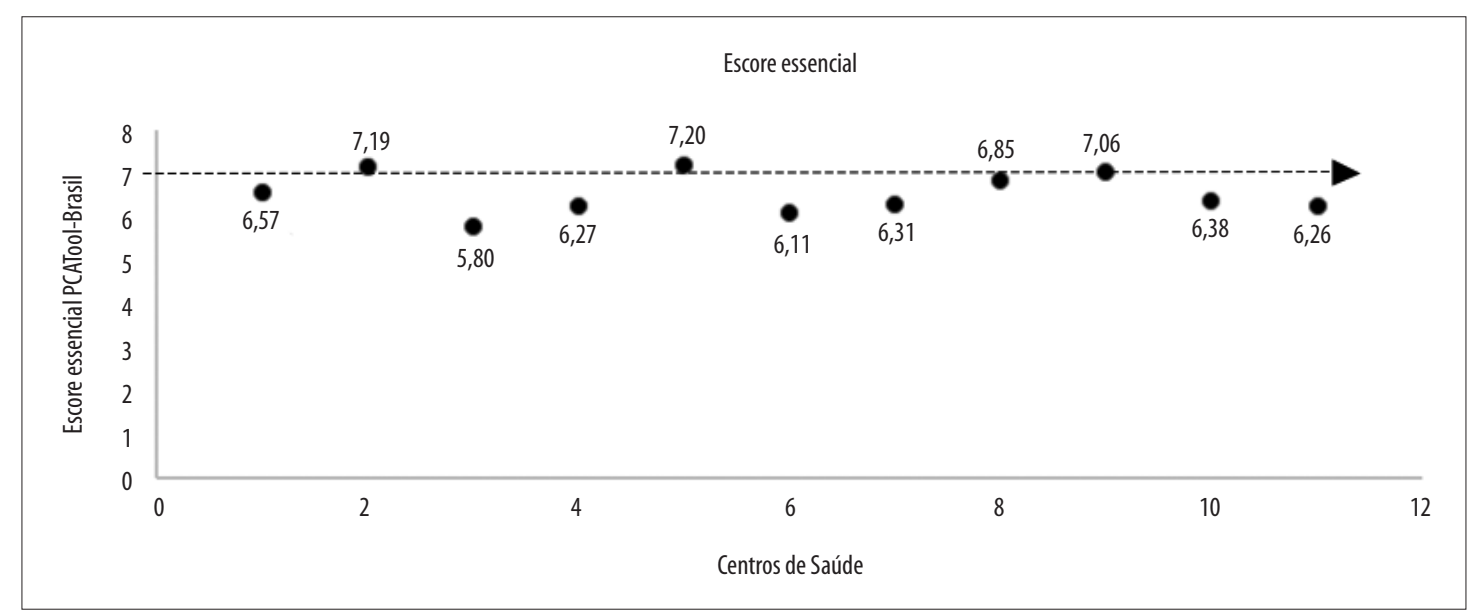

a) PCATool:Primary Care Assessment Tool.

Nota:

Os escores assumem valores de 0 a 10 .

Figura 2 - Escore médio essencial do PCATool-Brasila dos Centros de Saúde do Distrito Sanitário Norte, Florianópolis, Santa Catarina, 2012

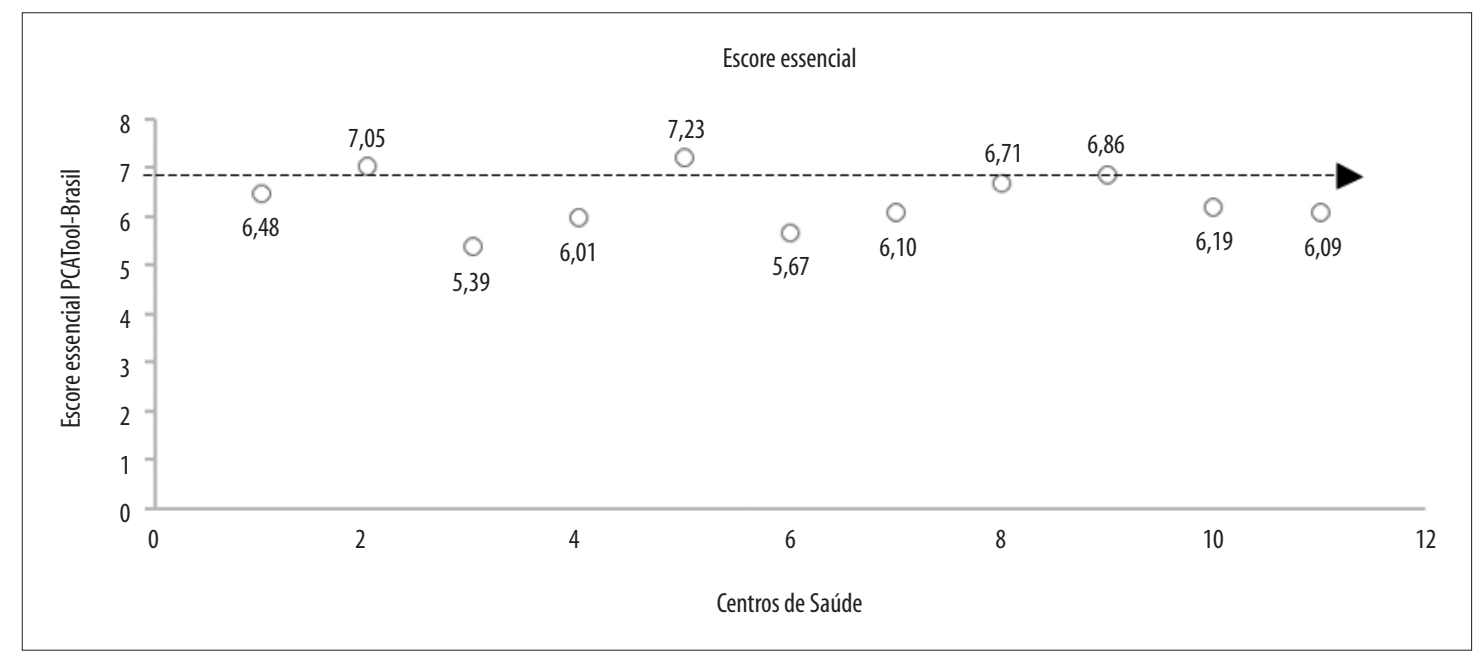

a) PCATool: Primary Care Assessment Tool.

Nota:

Os escores assumem valores de 0 a 10

\section{Figura 3 - Escore médio geral do PCATool-Brasila dos Centros de Saúde do Distrito Sanitário Norte, Florianópolis, Santa Catarina, 2012}

Quanto à Integralidade da atenção, tanto os serviços disponíveis (EG 5,4; $\left.\mathrm{IC}_{95 \%} 5,2 ; 5,7\right)$ quanto os serviços prestados (EG 5,1; $\left.\mathrm{IC}_{95 \%} 4,9 ; 5,3\right)$ obtiveram baixo escore. Semelhantemente concluíram Lima et al., depois de entrevistarem 215 usuárias entre 20 e 49 anos de idade, atendidas pela ESF de Serra, ES, (serviços disponíveis: EG 5,05; DP 1,6) (serviços prestados: EG 3,9; DP 2,16). ${ }^{25} 0$ baixo escore na Integralidade aponta a dificuldade dos serviços locais de saúde avaliados em compor arranjos na equipe de saúde capazes de garantir todos os serviços necessários para sua população, como também de se responsabilizarem pela oferta desses serviços em outros pontos da rede assistencial. ${ }^{24}$

Orientação Familiar (EG 5,8; $\mathrm{IC}_{95 \%}$ 5,5;6,1) e Orientação Comunitária (EG 5,2; $\left.\mathrm{IC}_{95 \%} 4,9 ; 5,5\right)$ obtiveram baixo escore geral de APS, resultados similares aos de Marques et al. ${ }^{26}$ quando avaliaram a APS e a saúde materno-infantil a partir de entrevistas com 76 cuidadores de 
crianças de 0-5 anos em uma comunidade quilombola de Minas Gerais (Orientação Familiar: EG 3,8; DP 2,6) (Orientação Comunitária: EG 5,8; DP 1,9) ${ }^{26}$ São evidências da dificuldade dos profissionais em dois aspectos relevantes: (i) ter um maior conhecimento acerca do funcionamento e da dinâmica familiar das pessoas, para a resolução de eventuais conflitos e necessidades, e (ii) saber reconhecer as necessidades da população em seus contextos físico, econômico e social. ${ }^{24}$

Entre as limitações do estudo em tela, a realização de até duas entrevistas por domicílio, contingenciada pela disponibilidade logística e financeira, pode ter elevado a média etária da amostra estudada e diminuído sua representatividade. Não obstante, a distribuição das frequências de idade da amostra estudada é semelhante à da população de adultos que, efetivamente, utilizou os serviços de APS em Florianópolis no ano de 2017. Embora os agentes comunitários/entrevistadores fossem alocados para outros centros de saúde que não os de sua atividade rotineira, no intuito de minimizar possíveis efeitos de viés de aferição, eles se apresentavam às pessoas como profissionais de saúde vinculados à prefeitura. Este fato pode ter intimidado os entrevistados, no momento de avaliarem seu serviço de referência como insatisfatório e, consequentemente, atribuídos melhores escores à avaliação, ainda que tenham participado voluntariamente e assinado o Termo de Consentimento Livre e Esclarecido para participação na pesquisa.

Outro possível limite do estudo se encontra na definição pelo PCATool-Brasil do mesmo peso a todos os atributos previstos no instrumento, supondo que o desempenho dos serviços pode ser avaliado exclusivamente pela presença $\mathrm{e}$ extensão desses atributos da APS. ${ }^{20}$ Outrossim, a aplicação do PCATool-Brasil limitou-se a usuários adultos. A não utilização de outras versões do instrumento - voltadas a crianças, profissionais ou gestores - pode levar a alguma restrição na generalização dos resultados de desempenho dos serviços avaliados, possivelmente evitada caso fossem investigados todos os envolvidos nos serviços.

\section{Referências}

1. Ministério da Saúde (BR). Secretaria de Atenção em Saúde. Departamento de Atenção Básica. Manual do instrumento de avaliação da atenção primária à saúde primary care assessment tool PCATool-Brasil [Internet]. Brasília: Ministério da Saúde; 2010 [citado 2017 ago 18] 80 p. Disponível em: http:// bvsms.saude.gov.br/bvs/publicacoes/manual_ avaliacao_pcatool_brasil.pdf
O desempenho dos serviços de APS avaliados deve ser aprimorado com o fortalecimento dos componentes de estrutura e processo, para seu melhor desempenho. 0 Acesso de Primeiro Contato/dimensão acessibilidade foi 0 atributo pior avaliado, contribuindo substancialmente para a diminuição dos escores médio geral e essencial. Logo, são necessárias mudanças no sentido de melhorar a acessibilidade a esses serviços. Alterações na forma de agendamento de consultas são importantes e podem contribuir nesse sentido.

Muitas equipes da ESF no país têm procurado melhorar sua acessibilidade implantando modelos mais ágeis de agendamento, como o chamado Acesso Avançado, que permite um maior equilíbrio entre a capacidade de oferta e a demanda do serviço de saúde, diminuindo o tempo de espera para consultas - não sem esforço, com as devidas adaptações à realidade local. ${ }^{27}$

Como conclusão deste relato, seus autores acreditam que o estabelecimento de estratégias de cooperação horizontal entre equipes e gestores de saúde, o apoio institucional ao planejamento, modo de condução do monitoramento e avaliação da presença e extensão dos atributos da APS, de forma periódica, e o uso racional de seus resultados, podem contribuir para a reformulação e desenvolvimento da qualidade da Atenção Primária à Saúde nos municípios brasileiros.

\section{Contribuição dos autores}

Vidal TB contribuiu na elaboração das versões preliminares e final do manuscrito. Vidal TB e Fontanive PVN contribuíram na concepção do estudo, coleta, análise e interpretação dos dados e revisão crítica do conteúdo. Tesser CD e Harzheim E contribuíram na concepção do estudo, elaboração de versões preliminares e revisão crítica do conteúdo. Todos os autores aprovaram a versão final do manuscrito e são responsáveis por todos os seus aspectos, incluindo a garantia de sua precisão e integridade.

2. Rowan MS, Lawson B, MacLean C, Burge F. Upholding the principles of primary care in preceptors' practices. Fam Med. 2002 NovDec;34(10):744-9.

3. Shi L, Starfield B, Xu J. Validating the Adult primary care assessment tool. J Fam Pract F. 2001 Feb;50(2):161-4. 
4. Clancy DE, Yeager DE, Huang P, Magruder KM Further evaluating the acceptability of group visits in an uninsured or inadequately insured patient population with uncontrolled type 2 diabetes. Diabetes Educ. 2007 Mar-Apr;33(2):309-14.

5. Pasarín MI, Berra S, Rajmil L, Solans M, Borrell C, Starfield B. An instrument to evaluate primary health care from the population perspective. Aten Primaria. 2007 Aug;39(8):395-401.

6. Tsai J, Shi L, Yu W-L, Lebrun LA. Usual source of care and the quality of medical care experiences a cross-sectional survey of patients from a taiwanese community. Med Care. 2010 Jul;48(7):628-34.

7. Berra S, Audisio Y, Mántaras J, Nicora V, Mamondi $\mathrm{V}$, Starfield B. Adaptación cultural y al sistema de salud argentino del conjunto de instrumentos para la evaluación de la atención primaria de la salud. Rev Argentina Salud Pública. 2011 sep;2(8):6-14.

8. Harzheim E, Oliveira MMC, Agostinho MR, Hauser L, Stein AT, Gonçalves MR, et al. Validação do instrumento de avaliação da atenção primária à saúde: PCATool-Brasil adultos. Rev Bras Med Fam Comunidade. 2013 out;8(29):274-84.

9. Harzheim E, Starfield B, Rajmil L, Álvarez-Dardet C, Stein AT. Internal consistency and reliability of primary care assessment tool (PCATool-Brasil) for child health services. Cad Saúde Pública. 2006 Aug;22(8):1649-59.

10. Stein AT. A avaliação dos serviços de saúde deve ser realizada com instrumentos validados. Epidemiol Serv Saúde. 2013 jan-mar;22(1):179-81.

11. Turci MA, Lima-Costa MF, Macinko J. Influência de fatores estruturais e organizacionais no desempenho da atenção primária à saúde em Belo Horizonte, Minas Gerais, Brasil, na avaliação de gestores e enfermeiros. Cad Saúde Pública. 2015 set;31(9):1941-52.

12. Chomatas E, Vigo A, Marty I, Hauser L, Harzheim E. Avaliação da presença e extensão dos atributos da atenção primária em Curitiba. Rev Bras Med Fam Comunidade. 2013 out;8(29):294-303.

13. Harzheim E, Pinto LF, Hauser L, Soranz D. Avaliação dos usuários crianças e adultos quanto ao grau de orientação para atenção primária à saúde na cidade do Rio de Janeiro, Brasil. Ciên Saúde Colet. 2016 maio;21(5):1399-408

14. Ministério da Saúde (BR). Índice de desempenho do sistema único de saúde [Internet]. 2012 [citado 2017 ago 21] . Disponível em: http://idsus.saude.gov.br/ index.html
15. Prefeitura Municipal (Florianópolis). Secretaria Municipal de Saúde. Portaria SS/GAB n 283, de 06 de agosto de 2007 . Aprova a política municipal de atenção a saúde, estabelecendo diretrizes e normas para a organização da atenção básica baseada na estratégia de saúde da família [Internet]. 2007 [citado 2018 ago 21]. 16 p. Disponível em: http://www.pmf.sc.gov.br/entidades/saude/index. php?cms $=$ saude $+\mathrm{d} a+$ familia\&menu $=5$

16. Instituto Brasileiro de Geografia e Estatística. Santa Catarina - Florianópolis - infográficos: evolução populacional e pirâmide etária [Internet]. 2010 [citado 2017 jun 28]. Disponível em: http://cod.ibge.gov.br/9D3

17. Prefeitura Municipal (Florianópolis). Secretaria Municipal de Saúde. População 2012 (rendimento/ raça) [Internet]. 2012 [citado 2017 ago 18]. Disponível em: http:/www.pmf.sc.gov.br/sistemas/saude/unidades_ saude/populacao/2012r/uls_2012_index.php

18. Prates ML, Machado JC, Silva LSD, Avelar PS, Prates LL, Mendonça ET, et al. Performance of primary health care according to PCATool instrument: a systematic review. Ciên Saúde Colet. 2017 Jun;22(6):1881-93.

19. Araújo LUA, Gama ZAS, Nascimento FLA, Oliveira HFV, Azevedo WM, Almeida Júnior HJB. Avaliação da qualidade da atenção primária à saúde sob a perspectiva do idoso. Ciên Saúde Colet. 2014 ago;19(8):3521-32.

20. Silva SA, Baitelo TC, Fracolli LA. Avaliação da atenção primária à saúde: a visão de usuários e profissionais sobre a estratégia de saúde da família. Rev Latino-Am Enfermagem. 2015 set-out;23(5):979-87.

21. Arakawa T, Arcêncio RA, Scatolin BE, Scatena LM, Ruffino-Netto A, Villa TCS. Acessibilidade ao tratamento de tuberculose: avaliação de desempenho de serviços de saúde. Rev Latino-Am Enfermagem. 2011 jul-ago;19(4):1-9.

22. Cunha EM, Giovanella L. Longitudinalidade/ continuidade do cuidado: identificando dimensões e variáveis para a avaliação da atenção primária no contexto do sistema público de saúde brasileiro. Ciên Saúde Colet. 2011;16(Suppl 1):1029-104.

23. Furtado MCC, Braz JC, Pina JC, Mello DF, Lima RAG. A avaliação da atenção à saúde de crianças com menos de um ano de idade na atenção primária. Rev LatinoAm Enfermagem. 2013 mar-abr;21(2):554-61.

24. Mendes EV. A construção social da atenção primária à saúde [Internet]. Brasília: Conselho Nacional de Secretários de Saúde - CONASS; 2015 [citado 2017 ago 7]. 194 p. Disponível em: http://www.conass.org.br/ biblioteca/pdf/A-CONSTR-SOC-ATEN-PRIM-SAUDE.pdf 
25. Lima EFA, Sousa AI, Primo CC, Leite FMC, Lima RCD, Maciel ELN. Avaliação dos atributos da atenção primária na perspectiva das usuárias que vivenciam o cuidado. Rev Latino-Am Enfermagem. 2015 maiojun;23(3):553-9.

26. Marques AS, Freitas DA, Leitão CDA, Oliveira SKM, Pereira MM, Caldeira AP. Atenção primária e saúde materno-infantil: a percepção de cuidadores em uma comunidade rural quilombola. Ciên Saúde Colet.

2014 fev;19(2):365-71.

\begin{abstract}
Objective: to evaluate the performance of Primary Health Care (PHC) in Florianopolis, SC, Brazil. Methods: this was a cross-sectional population-based study with adults living in the catchment areas of the Health Centers (HC) located in the Northern Health District of Florianópolis in 2012; the Primary Care Assessment Tool (PCATool-Brasil) was applied to assess the presence and extent of PHC attributes. Results: of the 598 interviewees, $68,4 \%$ reported that they considered PHC centers to be their reference service; while the utilization subdimension of the First Contact Access PHC attribute was the best evaluated (8.4;95\%CI8.2;8.6), the First ContactAccess/subdimension accessibility was the worst evaluated $(3.5 ; 95 \%$ CI $3.3 ; 3.6)$; four (36.6\%) of the eleven PHC centers evaluated had a high overall and essential PHC score, although their overall mean score was low (6.4; 95\%CI 6.2;6.5). Conclusion: considering the PHC model evaluated by PCATool-Brasil, PHC services need to be improved, especially with regard to their process and structure components.

Keywords: Primary Health Care; Family Health Strategy; Health Evaluation; Cross-Sectional Studies.
\end{abstract}

27. Wollmann A, Da Ros C, Lowen IMV, Moreira LR, Kami MT, Gomes MAG, et al. Novas possibilidades de organizar o acesso e a agenda na atenção primária à saúde [Internet]. Curitiba: Prefeitura Municipal de Curitiba; 2014 [citado 2017 ago 18]. 29 p. Disponível em: http://www.saude.curitiba.pr.gov.br/images/ cartilha acesso avançado 05_06_14.pdf

\section{Resumen}

Objetivo: evaluar el desempeño de la Atención Primaria a la Salud (APS) en Florianópolis, SC, Brasil. Métodos: estudio transversal de base poblacional realizado con adultos residentes en el territorio de los Centros de Salud (CS) del Distrito Sanitario Norte del municipio en 2012; el instrumento Primary Care Assessment Tool (PCATool-Brasil) fue aplicado para evaluar la presencia y la extensión de los atributos de la APS. Resultados: de 598 entrevistados, 68,4\% refirieron utilizar los CS como servicio de referencia; en el caso de que el Acceso de Primer Contacto/subdimensión utilización fue el atributo mejor valorado $\left(8,4 ; I_{95 \%}\right.$ 8,2;8,6), el Acceso de Primer Contacto/subdimensión accesibilidad, fue el peor $\left(3,5 ; I C_{95 \%} 3,3 ; 3,6\right)$; cuatro $(36,6 \%)$ de los once CS evaluados presentaron alto puntaje promedio esencial y general de APS, no obstante la puntuación promedio general de los mismos fuera baja $\left(6,4 ; I_{95 \%} 6,2 ; 6,5\right)$. Conclusión: considerando el modelo de APS evaluado por el PCATool-Brasil, los servicios de APS necesitan ser mejorados, especialmente en los componentes proceso y estructura.

Palabras clave: Atención Primaria de Salud; Estrategia de Salud Familiar; Evaluación en Salud; Estudios Transversales.

Recebido em 11/12/2017

Aprovado em 26/07/2018 